\title{
RECESIÓN EN LA OCUPACIÓN HOTELERA A PARTIR DE TRES ACONTECIMIENTOS: TERREMOTO, CIERRE DE AEROPUERTO, CONSTRUCCIÓN DE TRANVÍA. CASO CUENCA (ECUADOR)
}

\author{
Ana Lucía Serrano López* \\ Segundo Freire-Chaglla* \\ Universidad de Cuenca. Ecuador \\ Ileana Sanmartín-Rojas ${ }^{* *}$ \\ Universidad Nacional de Loja. Ecuador \\ Freddy Espinoza-Figueroa* \\ Universidad de Cuenca. Ecuador
}

\section{RESUMEN}

En Ecuador, el turismo representa la cuarta fuente de ingresos de los productos exportados, con un aporte de 1.433 millones de dólares a precios constantes y es equivalente al $1.5 \%$ de contribución al producto interno bruto para el año 2016². Para el estudio la Ocupación Hotelera se calculó de acuerdo a la Norma Eurostat 2012. La evaluación de los tres factores de la investigación, los resultados en la ocupación de alojamiento fueron los siguientes: 10\% menos en la ocupación por el cierre del aeropuerto, $6 \%$ menos por la ejecución de la red tranviaria y $3 \%$ menos por el terremoto. Con el primer evento (construcción del Tranvía) descendió al 28,1\%; con dos eventos (construcción del Tranvía y aeropuerto sin funcionamiento) al 22,2\%; y, cuando ocurren los tres eventos desciende al 17\%; por lo tanto, los eventos estudiados afectan negativamente la imagen del destino.

Palabras clave: Ocupación hotelera; terremoto; aeropuerto; Tranvía 4 Ríos; Eurostat; Cuenca-Ecuador.

Fecha de recepción: 12 de septiembre de 2017.

Fecha de aceptación: 16 de julio de 2018.

* Departamento de Investigación. Facultad de Ciencias de la Hospitalidad. Universidad de Cuenca. Calle Tarqui 13-46. Apartado postal 01.01.168. CUENCA (Ecuador).E-mail: ana.serrano@ucuenca.edu.ec, segundo. freire@ucuenca.edu.ec, freddy.espinoza@ucuenca.edu.ec

** Carrera de Turismo. Facultad Jurídica Social y Administrativa. Universidad Nacional de Loja. Av. Pío Jaramillo Alvarado y Reinaldo Espinosa. Casilla: S. LOJA (Ecuador).E-mail: ileana.sanmartin@unl.edu.ec

1 Elaboración propia acorde a información de estadísticas de comercio exterior de bienes y servicios emitidas por el Banco Central del Ecuador a agosto de 2017. 


\title{
Recession in the hotel occupation from three events: earthquake, airport closure, tramway construction. Case Cuenca (Ecuador)
}

\begin{abstract}
In Ecuador, tourism represents the fourth income source for exported products, with an unpot of 1433 million dollars at constant prices and it's the equivalent of $1.5 \%$ of the gross domestic product for the year 2016. For this study the Hotel occupation was calculated according to the Eurostat 2012 norm. The evaluation of the three research elements, the results in occupation and lodging were as it follows: $10 \%$ less occupation due to the closing of the airport, $6 \%$ reduction related to the construction of the tramway and $3 \%$ less due to the earthquake. With the first event (the construction of the Tramway) it went down to a 28.1\%; with two events (the construction of the Tramway and the construction of the airport) a $22.2 \%$ and with the three events it goes down to a $17 \%$; hence the studied events affect negatively the image of the destination.
\end{abstract}

Keywords: Hotel occupancy; earthquake; airport; Tranvía 4 Ríos; Eurostat; CuencaEcuador.

\section{INTRODUCCIÓN}

El impacto que ha ocasionado el turismo a nivel mundial -positivo y negativo- no podría ser posible sin el respaldo del alojamiento, uno de los servicios turísticos básicos que hace posible la permanencia de los visitantes en el destino.

El sector turístico es complejo debido a la diversidad de servicios que lo conforman y se convierta en una industria que enfrenta múltiples situaciones adversas (Peña y Serra, 2012). Produce impacto en la economía general de un país, al ser clave y fuentes de ingreso para la economía de un país (Romero, 2016) en dependencia de la madurez de los países en materia turística. Algunos países han sido sacudidos por distintos eventos que implican el decrecimiento de visitas y el desgaste del destino tales como: problemas políticos, desastres naturales, terrorismo, inseguridad, entre otros, creando escenarios de incertidumbre para los agentes turísticos y comprometiendo el éxito del destino (Melián, Fernández, y Hidalgo, 2014).

En el caso de Ecuador, el turismo representa la cuarta fuente de ingresos de los productos exportados, con un aporte de 1433 millones de dólares a precios constantes, atrás del petróleo, banano y camarones, y es equivalente al $1.5 \%$ de contribución al producto interno bruto para el año $2016^{2}$. Con basta razón el país planifica y ejecuta grandes proyectos turísticos que le ubiquen en un sitial importante en la generación de ingresos para el año 2018 (Castillo, Martínez, y Vázquez, 2016).

Cuenca, ciudad declarada Patrimonio Cultural de la Humanidad desde el 1 de diciembre de 1999 por la UNESCO (criterios ii, iii y v), ubicada a 450 kilómetros al sur de la

2 Información de elaboración propia acorde a información de estadísticas de comercio exterior de bienes y servicios emitidas por el Banco Central del Ecuador a agosto de 2017. 
línea equinoccial, también se ha visto afectada indirectamente por el terremoto del 16 abril del 2016 ocurrido en la provincia costera noroccidental de Manabí; además, ha debido enfrentar dificultades por la inestabilidad de los servicios aeroportuarios, cierre del aeropuerto, el cierre de calles y todas las molestias provocadas por los trabajos inconclusos de las obras del tranvía (Castillo L., 2017), que han disminuido el flujo de turistas, afectando principalmente al sector hotelero tradicional cuyo porcentaje de ocupación ha caído en un $30 \%$, según la Asociación Hotelera del Azuay que ha declarado al gremio en crisis (Marín, 2016). A esto se suma, la amenaza permanente de cerca de 160 plazas de alojamiento informal que no cumplen con los requisitos legales y compiten de manera desleal con los hoteles tradicionales de la ciudad (Castillo L., 2017).

Cuenca, con su promesa de convertirse en un destino turístico por excelencia en Ecuador y el reconocimiento como el destino número uno para jubilados por la Revista International Living en el año 2009, se ha visto afectada particularmente por tres factores que juegan un papel preponderante en la reducción de la ocupación hotelera, a saber: tranvía, aeropuerto y terremoto.

\subsection{Terremoto}

Los efectos causados por desastres naturales, van más allá de la destrucción de edificaciones e infraestructura, es decir se generan impactos a nivel social, cultural, económico e incluso medioambiental; el turismo no está exento de aquello, las percepciones de los turistas sobre un destino posterior a la catástrofe deterioran la imagen a largo plazo, dando lugar a una gran vulnerabilidad en el sistema turístico (Babinger, 2012).

Evidentemente la afluencia de turistas disminuye, no solo en el sitio donde se originó, sino en todo el país, puesto que el suceso genera pánico en los turistas, quienes prefieren renunciar a sus vacaciones hasta que se restablezcan las condiciones de acogida en los destinos; (López, Anato, y Rivas, 2004), esto, debido a que, naturalmente, los seres humanos, y por ende los turistas, rechazamos por instinto cualquier exposición a una vivencia de riesgo (Morillo, 2009).

El sismo ocurrido en el año 2004 en el Océano Índico, es considerado el terremoto más violento en el mundo desde 1964, el cual destruyó las playas de la región, especialmente de Tailandia, así como la infraestructura hotelera de la zona, lo que ocasionó una importante disminución de ingresos de turistas durante los años siguientes (López, Anato, y Rivas, 2004). En las playas de Chile, los constantes terremotos y tsunamis causas graves daños a las pequeñas localidades y sus negociantes que viven principalmente de la pesca y del turismo local (Morales, 2010).

En Ecuador, el terremoto de 7.8 grados en la escala de Richter ocurrido el 16 de abril de 2016, golpeó fuertemente al sector hotelero que hasta 2015 tenía una ocupación de hasta el 65,6\%, pero que después del desastre bajó a 57,2\% (Romero, 2016).

\subsection{Aeropuerto}

El turismo, por definición, según lo plantearon Hunziker y Krapf (1942) es el conjunto de relaciones producto del desplazamiento y la permanencia temporal de los visitantes en 
un lugar distinto al de su residencia habitual (Viloria, 2012). De ahí que, el turismo no sería posible sin el desplazamiento de las personas, y, por lo tanto, se debe entender que en este término está intrínsecamente vinculado el transporte para hacerlo posible (Viloria, 2012). De nada sirven los atractivos turísticos, las instalaciones y toda la infraestructura, sino se cuenta con los medios para trasladarse al sitio deseado (Hernández, 2008).

En el actual mundo globalizado, sin duda el transporte aéreo ha ganado un espacio trascendental gracias a la velocidad, seguridad y precio que lo han convertido en el medio de transporte más utilizado, no solo en el turismo, sino en los negocios en general, y representa una de las principales ventajas con que puede contar un destino turístico (Millán, 2010). En tal sentido, un aeropuerto constituye una infraestructura clave para que un destino turístico se conecte con el mundo y el mundo con el destino turístico (Vives, 2007), por ello, las ciudades turísticas se han visto favorecidas por la intensificación del transporte turístico desde finales del siglo XX y demandan cada vez más este medio de transporte para atraer visitantes (Maure, 2007).

Así, contar con un aeropuerto hace accesible un destino turístico y potencia sus cualidades como destino. Ejemplo de ello, es Puerto Vallarta y todo el territorio de Bahía Banderas, en México, que vio crecer sustancialmente la actividad turística con la construcción del aeropuerto, el cual también fue fundamental para el despegue del turismo en Acapulco (Cárdenas, 2014).

Por el contrario, cuando no existe la accesibilidad al destino, el turismo se detiene. Así lo señala Agostini (2012), quien indica que, entre los efectos del 11 de septiembre del 2001 en la economía de los Estados Unidos, el cierre del aeropuerto durante los días siguientes al atentado, implicó una reprogramación de los vuelos el cual generó pérdidas económicas bastante importantes.

En el caso de la ciudad de Cuenca, el cierre del Aeropuerto Mariscal Lamar debido al re-capeo de la pista que se realizaba en agosto del 2016 después de haberse reportado un accidentes en la pista ocasionado por la humedad en la pista el 28 de abril del 2016, afectó duramente al turismo de la ciudad, 148 vuelos fueron cancelados y más de 200 presentaron retrasos, lo que tuvo efecto en la reducción del movimiento de turistas hacia la ciudad, perjudicando al sector hotelero que durante ese tiempo no llegó ni al 30\% de ocupación, esto además de las múltiples cancelaciones de reservaciones que debieron enfrentar en una época especialmente importante para el sector como son las vacaciones de la Sierra (Castillo L., 2016).

\subsection{Tranvía}

Las obras de construcción que se realizan en los sitios de mayor afluencia turística, no solo causan molestias a los visitantes, sino que son determinantes a la hora de desistir de su viaje, afectando así, a los diferentes sectores que intervienen en el turismo, como el alojamiento. Tal es el caso del Hotel Miguel Ángel que se vio seriamente afectado por las obras de conexión de dos líneas del metro en Madrid, que provocaron que este hotel de cinco estrellas registró importantes pérdidas de clientela y, por tanto, de ingresos económicos; por lo que, la administración pública de Madrid debió indemnizar a su propietario por los perjuicios ocasionados (bajar los precios, reducir personal, cerrar zonas y estancias completas) (Salvador y Ramos, 2010). 
Como lo señala Rodríguez (2007) los proyectos de construcción de infraestructura en las ciudades deben responder eficientemente a las necesidades por las que fueron emprendidos, caso contrario, su fracaso afectará a los habitantes y todos aquellos sectores que vean involucrados en el proyecto, como es el caso de los establecimientos hoteleros. El desorden urbano provocado por interminables obras civiles llevadas a cabo en un sitio turístico incide negativamente en el desempeño de la actividad turística, reduce los flujos turísticos del área intervenida directamente por el proyecto, disminuye la eficacia económica y reduce la rentabilidad de las inversiones (Calderón y Orozco, 2009).

"Las calles de los centros históricos nunca fueron previstas para ser transitadas por coches y/o autobuses, al menos no de la forma intensiva como se da en la actualidad" (Cordero-Moscoso, 2012, p. 81). La construcción de un tranvía no solo sirve para mejorar las condiciones del sistema de transporte público, por defecto sirve para el turismo, ya sea porque lo pueden utilizar los visitantes para trasladarse de un sitio a otro, o porque su estructura permite que los visitantes puedan apreciar los atractivos del lugar durante su recorrido (Paúl y Pazos, 2009). Sin embargo, su construcción en Cuenca ha tomado más tiempo de lo planificado, es detenida o no encuentra fecha de culminación, se pierde el sentido de utilidad, crea molestias y ocasiona pérdidas económicas para la ciudad.

El crecimiento desordenado de la ciudad y la falta de cuidado en los trabajos de construcción ocasionados por el abandono o descuido gubernamental, afectan a los negocios en las zonas que han sido intervenidas para las obras en la ciudad (Sosa yCazal, 2014), entre ellos, los propietarios de alojamientos. En Cuenca, las demoras en la construcción del tranvía ejecutadas en pleno Centro Histórico desde noviembre de 2013, han afectado al sector comercial y al turismo, a tal punto que, según la Asociación Hotelera del Azuay en solo un año 14 alojamientos tuvieron que cerrar y la ocupación ha bajado hasta el $36 \%$ en el Centro Histórico y al $10 \%$ en los 18 alojamientos que se encuentran precisamente en la zona intervenida (Castillo L., 2017); las principales causas son las incomodidades que genera el cierre de las calles que impiden el libre desplazamiento de los visitantes, la contaminación auditiva provocada por el ruido de la maquinaria, la contaminación ambiental ocasionada por la cantidad de polvo que se produce, la dificultad para acceder a los locales comerciales, entre otros.

Los tres factores anteriores referidos, en parte han condicionado el ineficiente desarrollo del turismo de la ciudad, comprometiendo la imagen que se venía forjando como destino de calidad y que estaba ganando terreno entre los mejores destinos a nivel mundial, es decir, que estos factores externos implican un retroceso en su desarrollo. Resulta importante considerar que la planeación del espacio público, en ciudades con potencial turístico, deben enfocarse en un modelo que beneficie al ciudadano sin olvidar el turismo con funcionalidad y operatividad (Sosa yCazal, 2014). De igual forma, los planes de recuperación luego del terremoto deben tener muy en cuenta, no solo a las grandes cadenas hoteleras, sino también a los pequeños y medianos propietarios de servicios turísticos (Cajas yAlberto, 2016), tanto en la zona afectada como en el resto del país, tomando lo que ha pasado como referencia para empezar a dedicar recursos a la gestión de crisis. 


\section{MATERIALES Y MÉTODOS}

El presente estudio se formuló en dos momentos; el primero se refiere a la recopilación y clasificación de información en los periodos que aparecen los efectos de las variables; y, en el segundo momento, comparaciones entre un momento inicial sin afectación y los eventos suscitados como factores de análisis (las obras de construcción del Tranvía 4 Ríos, el período de cierre del aeropuerto Mariscal Lamar, el Terremoto de Manabí).

La muestra estuvo compuesta por información perteneciente a 109 establecimientos de alojamiento de Cuenca de los cuales y según la anterior categorización de la Ley de Turismo, 15 son considerados de lujo, 19 de primera clase, 22 de segunda, 24 de tercera y 29 de cuarta. A cada uno se solicitó los registros ocupacionales de 19 meses comprendidos entre junio (2015) y diciembre (2016). En promedio 16 establecimientos no reportaron por lo menos un dato al mes, en tal razón, la muestra mensual de los 109 establecimientos en 19 meses asciende a 2055 registros de huéspedes (Tabla 1).

\section{Tabla 1 \\ ESTABLECIMIENTOS DE HOSPEDAJE EVALUADOS}

\begin{tabular}{lcc}
\hline Periodo & Establecimientos & Registros \\
\hline Junio-agosto 2015 & 109 & 327 \\
Septiembre-diciembre 2015 & 109 & 434 \\
Enero-abril 2016 & 109 & 433 \\
Mayo-Agosto 2016 & 109 & 431 \\
Septiembre-diciembre 2016 & 109 & 430 \\
\hline Total & 109 & 2055 \\
\hline
\end{tabular}

Para el cálculo de ocupación hotelera se utilizó la tasa de ocupación Euro Stats, sistema que proporciona estadísticas a nivel europeo sobre la ocupación de establecimientos de alojamiento turístico y la demanda turística. La fórmula de cálculo para obtener la tasa de ocupación utilizado por Eurostats implica la multiplicación del número de huéspedes por la cantidad de noches que se hospedaron sobre el número de plazas por cada día del mes (Eurostat, 2012); a continuación, se expresa la ecuación empleada:

\section{Ecuación 1 \\ OCUPACIÓN HOTELERA USADA POR EURO STATS}

Ocupación hotelera $=\frac{(\text { Huéspedes nacionales } \times \text { úmero de noches })+(\text { Huéspedes extranjeros } \times \text { Número de noches })}{N \text { úmero de plazas } \times \text { úmero de días del mes }}$

Los resultados se procesaron en el Software SPSS 22. Se emplea la prueba no paramétrica denominada Kruskal Wallis para comprobar la asociación de los factores sobre la 
tasa de ocupación hotelera, así como, para especificar cuáles son estos factores, se emplea el estadístico de prueba U de Mann Whitney. El nivel de significancia establecido fue de 0,05 . Ello implicó que si el p valor alcance 0.05 , se acepta la hipótesis alternativa de asociación de variables, de lo contrario se la descarta.

\section{RESULTADOS}

La presencia de los tres factores ha generado cambios importantes en el comportamiento de la tasa de ocupación hotelera. En efecto, antes de la construcción del Tranvía 4 Ríos se advierte que la tasa de ocupación hotelera fue del $31 \%$, con el proyecto en marcha se redujo al $25 \%$. Cuando se cierra el aeropuerto la tasa de ocupación hotelera se redujo al $19 \%$, mientras que, en el trimestre inmediato al terremoto, la tasa llega al nivel más bajo que es del 17\%. En todos los casos, se advierten diferencias significativas a juzgar por los resultados de la prueba U de Mann Whitney (Tabla 2).

Tabla 2

DESCRIPCIÓN DE LA OCUPACIÓN HOTELERA SEGÚN FACTORES

\begin{tabular}{|c|c|c|c|c|c|c|c|}
\hline \multicolumn{2}{|l|}{ Factores } & $\mathbf{n}$ & $\begin{array}{l}\text { Media de } \\
\text { ocupación }\end{array}$ & $\begin{array}{c}\text { Desviación } \\
\text { estándar }\end{array}$ & $\mathrm{U}$ & $\mathrm{Z}$ & $\mathrm{P}$ \\
\hline \multirow{2}{*}{ Tranvía } & Sin construcción ${ }^{\mathrm{a}}$ & 349 & 30,9 & 26,2 & \multirow{2}{*}{$259.395,5$} & \multirow{2}{*}{$-3,792$} & \multirow{2}{*}{0,000} \\
\hline & Con construcción ${ }^{b}$ & 1706 & 25,1 & 23,5 & & & \\
\hline \multirow{2}{*}{ Aeropuerto } & En funcionamiento & 1497 & 28,7 & 24,9 & \multirow{2}{*}{$314.740,0$} & \multirow{2}{*}{$-8,604$} & \multirow{2}{*}{0,000} \\
\hline & Sin funcionamiento ${ }^{c}$ & 558 & 18,9 & 20,1 & & & \\
\hline \multirow{2}{*}{ Terremoto } & Sin terremoto & 1701 & 20,0 & 24,4 & \multirow{2}{*}{$206.770,5$} & \multirow{2}{*}{$-9,285$} & \multirow{2}{*}{0,000} \\
\hline & Trimestre terremoto & 354 & 17,0 & 20,1 & & & \\
\hline
\end{tabular}

$\mathrm{n}=$ muestra investigada; $\mathrm{U}=\mathrm{U}$ de Mann Whitney; $\mathrm{Z}=$ Análisis de normalidad; $\mathrm{p}=$ nivel de significancia. a. julio-diciembre 2015, b. enero-diciembre 2016, c. abril-agosto 2016, d. abril-junio 2016

En lo que respecta a la combinación de factores, se advierte cómo se va reduciendo el nivel de la tasa de ocupación hotelera a medida que se agudizan los problemas. De esta manera, cuando ninguno de los factores había sucedido (ausencia), la ocupación era del $31 \%$, cuando sucedió un factor descendió al 28\%, cuando sucedieron dos factores al $22 \%$ y cuando ocurrieron todos los tres descendió al 17\% (Tabla 3).

Para establecer diferencias específicas relativas a la combinación de factores, se aplicó la prueba de Kruskal Wallis misma que dio un resultado de diferencias significativas, sin embargo, para especificar cuáles son estas diferencias, se aplicó la prueba U de Mann Whitney. Al respecto, se encontró que, con excepción del primer trimestre de construcción del tranvía comparado con la no construcción del mismo, en todas las demás comparaciones, existen diferencias significativas (Véase Tabla 4) en las cuales el p valor es menor que 0,05 . 
Tabla 3

DESCRIPCIÓN DE LA OCUPACIÓN HOTELERA SEGÚN COMBINACIÓN DE FACTORES

\begin{tabular}{|c|c|c|c|c|c|}
\hline Tranvía & Aeropuerto & Terremoto & $\begin{array}{c}\text { Media de } \\
\text { ocupación }\end{array}$ & $\begin{array}{c}\text { Desviación } \\
\text { estándar }\end{array}$ & $\mathbf{n}$ \\
\hline \multirow{4}{*}{ Ausencia } & Funcionando & \multirow{2}{*}{$\begin{array}{l}\text { Sin terremoto } \\
\text { Total }\end{array}$} & 30,9 & 26,2 & 349 \\
\hline & & & 30,9 & 26,2 & 349 \\
\hline & Total & Sin terremoto & 30,9 & 26,2 & 349 \\
\hline & & Total & 30,9 & 26,2 & 349 \\
\hline \multirow{8}{*}{ Construcción } & Funcionando & \multirow{2}{*}{$\begin{array}{l}\text { Sin terremoto } \\
\text { Total }\end{array}$} & 28,1 & 24,5 & 1148 \\
\hline & & & 28,1 & 24,5 & 1148 \\
\hline & Sin funcionar & \multirow{3}{*}{$\begin{array}{l}\text { Sin terremoto } \\
\text { Trimestre terremoto } \\
\text { Total } \\
\end{array}$} & 22,2 & 19,8 & 204 \\
\hline & & & 17,0 & 20,1 & 354 \\
\hline & & & 18,9 & 20,1 & 558 \\
\hline & Total & \multirow{3}{*}{$\begin{array}{l}\text { Sin terremoto } \\
\text { Trimestre terremoto } \\
\text { Total }\end{array}$} & 27,2 & 23,9 & 1352 \\
\hline & & & 17,0 & 20,1 & 354 \\
\hline & & & 25,1 & 23,5 & 1706 \\
\hline \multirow{8}{*}{ Total } & Funcionando & \multirow{2}{*}{$\begin{array}{l}\text { Sin terremoto } \\
\text { Total }\end{array}$} & 28,7 & 24,9 & 1497 \\
\hline & & & 28,7 & 24,9 & 1497 \\
\hline & Sin funcionar & \multirow{3}{*}{$\begin{array}{l}\text { Sin terremoto } \\
\text { Trimestre terremoto } \\
\text { Total } \\
\end{array}$} & 22,2 & 19,8 & 204 \\
\hline & & & 17,0 & 20,1 & 354 \\
\hline & & & 18,9 & 20,1 & 558 \\
\hline & Total & \multirow{3}{*}{$\begin{array}{l}\text { Sin terremoto } \\
\text { Trimestre terremoto } \\
\text { Total }\end{array}$} & 28,0 & 24,4 & 1701 \\
\hline & & & 17,0 & 20,1 & 354 \\
\hline & & & 26,1 & 24,1 & 2055 \\
\hline
\end{tabular}

Tabla 4

DIFERENCIAS DE ACUERDO A FACTORES COMBINADOS

\begin{tabular}{|l|l|c|c|c|}
\hline & & $\mathbf{U}$ & $\mathbf{Z}$ & $\mathbf{p}$ \\
\hline \multirow{4}{*}{ 0 Sin tranvía } & 1 Primer trimestre construcción del tranvía & $189.442,5$ & $-1,539$ & 0,124 \\
\cline { 2 - 5 } & $\begin{array}{l}\text { 2 Segundo cuatrimestre de construcción de } \\
\text { tranvía y sin aeropuerto }\end{array}$ & $29.910,0$ & $-3,137$ & 0,002 \\
\cline { 2 - 5 } & $\begin{array}{l}\text { 3 Construcción del tranvía, sin aeropuerto y } \\
\text { trimestre terremoto }\end{array}$ & $40.043,0$ & $-8,072$ & 0,000 \\
\hline \multirow{3}{*}{$\begin{array}{l}\text { 1 Primer trimestre } \\
\text { construcción del } \\
\text { tranvía }\end{array}$} & 0 Sin tranvía & $18.9442,5$ & $-1,539$ & 0,124 \\
\cline { 2 - 5 } & $\begin{array}{l}\text { 2 Segundo cuatrimestre de construcción de } \\
\text { tranvía y sin aeropuerto }\end{array}$ & $10.5269,0$ & $-2,302$ & 0,021 \\
\cline { 2 - 5 } & $\begin{array}{l}\text { 3 Construcción del tranvía, sin aeropuerto y } \\
\text { trimestre terremoto }\end{array}$ & $13.9518,0$ & $-8,926$ & 0,000 \\
\hline
\end{tabular}




\begin{tabular}{|l|l|c|c|c|}
\hline $\begin{array}{l}2 \text { Segundo } \\
\text { cuatrimestre de } \\
\text { construcción } \\
\text { de tranvía y sin } \\
\text { aeropuerto }\end{array}$ & 0 Sin tranvía & $29.910,0$ & $-3,137$ & 0,002 \\
\cline { 2 - 5 } & 1 Primer trimestre construcción del tranvía & $105.269,0$ & $-2,302$ & 0,021 \\
\cline { 2 - 5 } & $\begin{array}{l}\text { 3 Construcción del tranvía, sin aeropuerto y } \\
\text { trimestre terremoto }\end{array}$ & $27.209,5$ & $-4,852$ & 0,000 \\
\hline \multirow{2}{*}{$\begin{array}{l}\text { 3 Construcción } \\
\text { del tranvía, sin } \\
\text { aeropuerto y } \\
\text { trimestre terremoto }\end{array}$} & 0 Sin tranvía & $40.043,0$ & $-8,072$ & 0,000 \\
\cline { 2 - 5 } & $\begin{array}{l}\text { 1 Srimer trimestre construcción del tranvía } \\
\text { tranvía y sin aeropuerto }\end{array}$ & $139.518,0$ & $-8,926$ & 0,000 \\
\hline
\end{tabular}

La ilustración 1 muestra barras de error relativas al comportamiento dispar de la ocupación hotelera según la acumulación de los factores y resume aquello que se ha señalado en las tablas 3 y 4 . La barra correspondiente a sin problema y construcción del tranvía (primer trimestre de construcción), se muestran en un nivel parecido, no obstante, en las demás barras de advierten mayores distancias. En ellas se advierte que, a mayor número de factores, menor es la tasa de ocupación hotelera.

\section{Ilustración 1 \\ BARRAS DE ERROR}

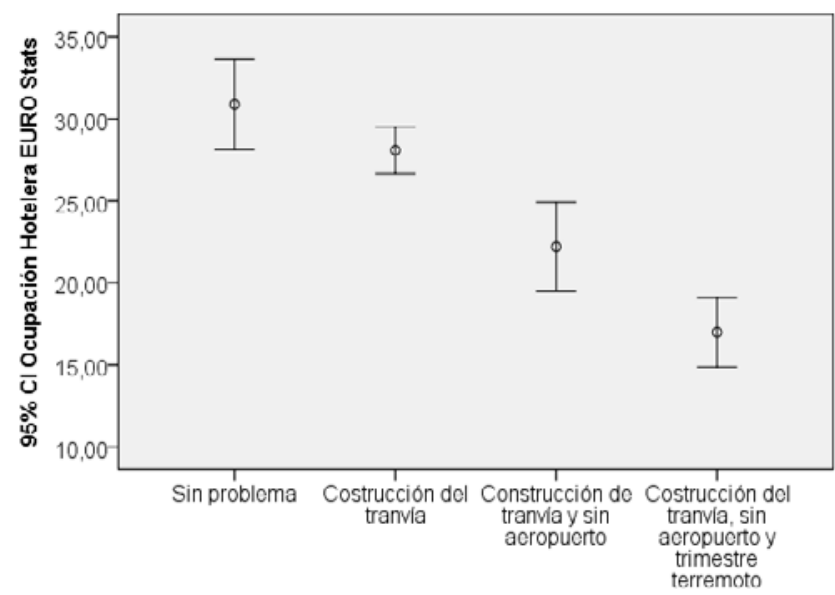

Eventos

\section{DISCUSIÓN}

El turismo es particularmente sensible a distintos acontecimientos. Es imprescindible que los destinos sepan cómo gestionarlo en situaciones de crisis a sabiendas de la vulnerabilidad del ser humano ante desastres naturales e incluso acciones antrópicas.

Dos eventos grandes -los ataques terroristas del 11-S y el tsunami asiático de 2004tuvieron una gestión de crisis del turismo reactiva, no obstante, en un contexto de gestión de servicios es necesario una gestión proactiva, siendo imperante medidas previas ante 
eventos de crisis, siendo el comienzo real de cualquier respuesta estratégica para la recuperación (PforryHosie, 2008). Estos incidentes pueden destruir la imagen que se tiene del destino (Hanno, Feldesz, y Merten, 2016).

Los eventos antrópicos objeto de este documento -cierre del aeropuerto y construcción del Tranvía- también merecen respuestas proactivas. Button, Lall, Stough, y Trice (1999), Brueckner (2003), y, Bel y Fageda (2008) sostienen que "[...]los aeropuertos juegan un papel fundamental en el desarrollo económico del territorio donde se ubican" (Bel y Fageda, 2011, p. 110), sin embargo, muchos problemas se generan a causa del modelo de gestión centralizado o administrado por el sector público. En el año 2011 España propuso una reforma a los modelos de gestión de los aeropuertos. Bel y Fageda (2011) sostuvieron que se debía emplear una "gestión individualizada del sistema aeroportuario [...] [para] introducir competencia entre los aeropuertos y mejorar la eficiencia general del sistema [...] la individualización es compatible con el diseño de mecanismos de subsidio para los aeropuertos más pequeños que sean más transparentes y eficientes en su aplicación, y más eficaces para el logro de sus objetivos" (p. 127). Por otro lado, en Perú existen opciones como: i) la infraestructura más grande, el Aeropuerto Internacional Jorge Calvache aporta con sus ingresos para financiar la expansión de infraestructura en todo el país, ii) alianzas público-privadas para la gestión, y, iii) concesiones; en cualquiera de los casos lo ideal es liberar presupuesto público, olvidando la idea recurrente de regresar a los subsidios cruzados sectoriales (Barrantes Cáceres, 2012).

Los tranvías como medio de transporte urbano volvieron a tener auge a finales del siglo XX, tal es el caso de Estados Unidos (década de 1980s), España (década de 1990s) (Albertos Puebla, Salom Carrasco, Pitarch Garrido, Noguera Tur, y Maestro Cano, 2010), como opción para revitalizar ciudades y como soluciones de movilidad, no obstante, la implementación de estas mega-obras resulta un gran problema, más aún cuando la planificación no es acorde a los recursos; la inversión en este tipo de proyectos es alta, las rutas son inflexibles, sin embargo puede resultar un buen elemento para reducir la contaminación y ser un elemento destacado para el desarrollo del turismo, siempre y cuando se planifiquen acciones integrales en torno al sistema de transporte urbano (Moscoso-Cordero, 2012); en cualquier caso es fundamental la gestión de crisis en el aspecto turístico; en el caso de Cuenca parece ser lejana debido al obsoleto modelo de gestión turística existente que tiene traspasadas las competencias de turismo, no obstante se ejecuta solamente lo concerniente a promoción.

Como cualquier aspecto que infiere en la economía, la industria de alojamiento debe hacer frente a variables que están fuera de su control y que afectan fuertemente a sus ingresos. Resulta importante identificar estos factores y su impacto para crear una fuente de información como una oportunidad para crear anticipadamente planes de contingencia que a futuro permitan a los propietarios estar mejor preparados para reducir el impacto. En tal sentido, los resultados encontrados en este estudio, muestran que el destino Cuenca, en cuanto a los índices de ocupación hotelera, indudablemente se ha visto afectado con la ocurrencia de los tres acontecimientos analizados.

Así, los datos evidencian que, con respecto a los trabajos de construcción del Tranvía, iniciados en noviembre del 2013, -antes de su construcción- la tasa hotelera fué del 31\%, mientras que con el proyecto en marcha se redujo al $25 \%$, cifra muy inferior a las previ- 
siones del 36\% establecidas por la Asociación Hotelera del Azuay en el Centro Histórico de la ciudad de Cuenca, lugar donde se concentra el $61 \%$ de sitios de alojamiento. Al respecto, Calderón y Orozco (2009) advierten que los desórdenes urbanos provocados por obras civiles inconclusas afectan al sitio turístico porque impiden el normal desarrollo de la actividad turística, y en efecto, reduce el flujo de turistas, y por supuesto, el ingreso de dinero.

En el caso del cierre del aeropuerto "Mariscal Lamar", iniciado en agosto de 2016, los resultados han demostrado que la tasa de ocupación hotelera se redujo del 29\% al 19\%, debido tanto al cierre, como a las cancelaciones imprevistas de los vuelos y los continuos atrasos por revisiones de la Dirección General de Aviación Civil, quienes buscaban garantizar seguridad en los vuelos. La situación del aeropuerto no solo afectó al ingreso de turistas, también generó un ambiente de desconfianza porque las aerolíneas continuaban emitiendo pasajes, siendo estos cancelados a última hora, lo cual comprometió la imagen de la ciudad, y, del sistema de transporte aéreo de la misma, proyectándose como un destino turístico desorganizado y poco respetuoso con el turista. Esta situación ha sido seguramente el golpe más duro para la ciudad, $10 \%$ menos a la ocupación anterior al suceso, mientras que a causa del Tranvía fue $6 \%$ menos y por el terremoto $3 \%$ menos; de nada sirven los atractivos turísticos, las instalaciones y toda la infraestructura, si el turista no cuenta con los medios para trasladarse (Hernández, 2008), el turismo está intrínsecamente vinculado el transporte para hacerlo posible (Viloria, 2012).

En cuanto al tercer suceso, se ha demostrado que los efectos del terremoto ocurrido en abril 2016 provocaron una reducción del 20\% de ocupación al 17\%. En este sentido, se puede inferir que la baja de $3 \%$ en ocupación, es considerablemente menor a lo sucedido en el epicentro -Provincia de Manabí- experimentando una reducción de hasta el $50 \%$ de su capacidad hotelera (Romero, 2016). En Cuenca el terremoto indirectamente tuvo efectos negativos en el sector hotelero, es inevitable que después de una catástrofe la afluencia de turistas disminuye, no solo en el sitio donde se originó, sino en todo el país, ya que el suceso genera pánico en los turistas, quienes prefieren renunciar a sus motivaciones de viaje hasta que se restablezcan las condiciones de acogida en los destinos (López, Anato y Rivas, 2004).

Después de haber atravesado algunos años de favorable crecimiento, especialmente porque Cuenca empezaba a darse a conocer al mundo como un destino turístico prometedor, a partir de la construcción del Tranvía en el año 2013 los indicadores de ocupación hotelera han empezado a mostrar signos de debilitamiento a medida que los problemas se agudizan (entre junio del año 2015 y diciembre del año 2016) la afluencia total de turistas disminuyó del 30,9\% al 17\% de acuerdo a la media de ocupación hotelera observado en los resultados expuestos (Tabla 3 ).

En este contexto, cuando ninguno de los tres eventos distorsionadores (o de efecto) había sucedido, la ocupación hotelera era del 30,9\%; con el primer evento (construcción del Tranvía) descendió al 28,1\%; con dos eventos (construcción del Tranvía y aeropuerto sin funcionamiento) al 22,2\%; y, cuando ocurren los tres eventos desciende al $17 \%$. Se puede observar que la situación se complica cuando se suman eventos externos que están fuera del control de sus propietarios (Melián, Fernández, y Hidalgo, 2014). 
Estos factores de cambio en el servicio de alojamiento, ponen en una encrucijada a los servicios de alojamiento, pues corren el riesgo de desaparecer del mercado (Wallingre, 2005), situación que se complica cuando se suman factores externos que están fuera del control de sus propietarios (Melián, Fernández, y Hidalgo, 2014), sin embargo, no todos los elementos inciden de la misma manera e intensidad en el comportamiento de la demanda turística (Jiménez, Peña, y Ruiz, 2015); el transporte ha sido el más afectado, puesto que tiene un porcentaje mayor de diferencia entre la ocupación anterior a la construcción y después del suceso, en relación con los otros dos acontecimientos.

\section{CONCLUSIONES}

Un comportamiento común es dar por sobreentendido que el turismo es posible en cualquier condición y que siempre van a existir turistas, olvidando que gestionar el turismo es gestionar la complejidad en base a datos, información, pensamiento y conocimiento, olvidando considerar que turismo es personas (turistas) y sin estas no hay turismo (Capece, 2012). La imagen de un destino cumple una importante función porque los potenciales turistas no tienen más que la imagen para decidir ir o no ir a un destino, ocupar o no un alojamiento. Los factores estudiados como construcción del tranvía, cierre del aeropuerto y trimestre inmediato al terremoto, afectan a la formación de la imagen en los turistas. Por lo tanto, Cuenca se encuentra en una etapa crítica dentro de su desarrollo como destino turístico, especialmente en materia de alojamiento en los tres trimestres del año 2016 contabilizados a partir de la aparición de esos factores. El presente estudio, ha demostrado que la acumulación de factores genera una disminución significativa en la ocupación hotelera.

\section{BIBLIOGRAFÍA}

AGOSTINI, C. (2012): «El mercado de transporte aéreo: lecciones de política de una revisión de la literatura», Journal of Transport Literature, vol. VI, n 3, pp. 239-277.

BABINGER, F. (2012): «El turismo ante el reto de peligros naturales recurrentes: una visión desde Cancún», Investigaciones Geográficas, $n^{\circ}$ 78, pp. 75-88.

BANKS, L. (2009): «La Paz a prueba de un siniestro», Revista Apetito, n 6, pp. 22-23.

BARRANTES CÁCERES, R. (2012): «Desarrollo de la Infraestructura de Aeropuertos en el Perú», Círculo de Derecho Administrativo, n 12, pp. 209-220.

BEL, G. y FAGEDA, X. (2008): «Getting There Fast: Globalization, Intercontinental Flights and Location of Headquarters», Journal of Economic Geography, n 8, pp. 471-495.

BEL, G. y FAGEDA, X. (2011): «La reforma del modelo de gestión de aeropuertos en España: ¿Gestión conjunta o individual?», Revista de Economía Pública, vol. I, nº 196, pp. 109-130.

BRUECKNER, J.K. (2003): «Airline traffic and urban economic development», Urban Studies, $\mathrm{n}^{\circ}$ 40, pp. 1.455-1.469.

BUTTON, K., LALL, S., STOUGH, R. y TRICE, M. (1999): «High-technology employment and hub airports», Journal of Air Transport Management, $\mathrm{n}^{\circ}$ 5, pp. 53-59. 
CAJAS, J. y ALBERTO, A. (2016): «Pérdidas, respuestas y dudas de una tragedia: Reflexiones después del terremoto de abril 16 del 2016», Rebelión, n 6.

ÇAKAR, K. (2018). «Critical success factors for tourist destination governance in times of crisis: a case study of Antalya, Turkey». Journal of Travel y Tourism Marketing, pp. 1-17.

CALDERÓN, J. y OROZCO, M. (2009): «Planeación y modelo urbano: el caso de Cancún, Quintana Roo», Quivera, vol. XI, n² 2, pp. 18-34.

CAPECE, G. (2012): Turismo: Gestión de la complejidad. Buenos Aires, Fundación.

CÁRDENAS, E. (2014): «El aeropuerto de Puerto Vallarta: factor de impulso del turismo nacional e internacional», Cuadernos de Turismo, n 14, pp. 53-68.

CASTILLO, E., MARTÍNEZ, F. y VÁZQUEZ, E. (2016): «El turismo en Ecuador, nuevas tendencias en el turismo sostenible y contribución al crecimiento económico», Revista Galega de Economía, vol. XXIV, n² 2, pp. 69-88.

CASTILlO, L. (5 de septiembre de 2016): El cierre del aeropuerto afecta más al turismo. El Comercio.

CASTILlO, L. (Enero de 2017): Un éxodo del centro de Cuenca por el tranvía. El Comercio.

EUROSTAT. (2012): Methodological manual for tourism statistics Versión 1.2. European Union, Eurostat European Commision.

GRANADOS, M. (2000): «Rol de los municipios en el desarrollo del turismo», Gestión en el Tercer Milenio, vol. III, $\mathrm{n}^{\circ}$ 6, pp. 51-72.

HANNO, M. M., FELDESZ, K. y MERTEN, P. (2016): «Crisis Management in Tourism A Literature Based Approach on the Proactive Prediction of a Crisis and the Implementation of Prevention Measures», Athens Journal of Tourism, vol. III, n 2, pp. 89-102.

HERNÁNDEZ, J. (2008): «Turismo de masas y transporte: el gran reto del turismo del Siglo XXI», Scripta Nova, vol. XII, n 258, pp. 256-280.

JIMÉNEZ, A. (2008): «Las cadenas hoteleras en el mundo y evolución de su operación en México al inicio del siglo XXI», Innovar, vol. XVII, n³ 32, pp. 167-194.

LÓPEZ, M., ANATO, M. y RIVAS, B. (2004): «Impacto de los acontecimientos mundiales en el turismo. Casos de estudio», Economía, n 19 , pp. 135-165.

MARÍN, J. (5 de junio de 2016): La Asociación Hotelera de Cuenca se declara en crisis. Expreso.

MAURE, G. (2007): «Definiciones y tendencias del turismo de eventos», Contribuciones a la Economía, $\mathrm{n}^{\circ} 82$.

MELIÁN, L., FERNÁNDEZ, M. e HIDALGO, M. (2014): «El sector turístico en contextos de crisis», Universia Business Review, vol. 83, n 10, pp. 110-129.

MENOYA, S., GÓMEZ, G. y PÉREZ, I. (2017): «Modelo basado en enfoque de cadena de valor para gestión del turismo desde el gobierno local en municipios con vocación turística», Retos de la Dirección, vol. XI, n 1, pp. 172-204.

MILlÁN, M. (2010): «Planificación: Transportes, turismo y territorio», Gran Tour: Revista de Investigaciones Turísticas, $\mathrm{n}^{\circ} 1$, pp. 97-119.

MORALES, R. (2010): «Terremoto y tsunami del 27 de febrero de 2010, efectos urbanos en localidades de la provincia de Arauco», Urbano, vol. XII, n² 22, pp. 43-62. 
MORILLO, M. (2009): «Capacidad de resistencia del turismo en épocas de crisis, Revista Saber, vol. XXI, n 3, pp. 292-305.

MOSCOSO, M.S. (2012): «Los vehículos motorizados privados y el problema de transporte público en los centros históricos: el caso de Cuenca-Ecuador», Estoa. Revista de La Facultad de Arquitectura Y Urbanismo de La Universidad de Cuenca, $\mathrm{n}^{\circ} 1$, pp. 79-93. Disponible en http://dspace.ucuenca.edu.ec/bitstream/123456789/27615/1/ Estoa1_9.pdf

NAVAS, M. y FERMÍN, O. (2011): «Conflictos de control y competencia en la actividad turística-recreacional», Gestión Turística, n 16, pp. 127-136.

PASCARELLA, R. y FONTES, J. (2010): «Competitividad de los destinos turísticos. Modelo de evaluación basado en las capacidades dinámicas y sus implicancias en las políticas públicas», Estudios y perspectivas en turismo, vol. XIX, n 1, pp. 1-17.

PAÚL, V. y PAZOS, M. (2009): «El transporte como producto turístico en Galicia: un análisis de la oferta actual», Revista Galega de Economía, vol. XVII, n² 2, pp. 1-29.

PEÑA, D. y SERRA, A. (2012): «Responsabilidad social empresarial en el sector turístico», Estudios y Perspectivas en Turismo, n² 21, pp. 1456-1480.

PFORR, C. y HOSIE, P.J. (2008): «Crisis Management in Tourism», Journal of Travel y Tourism Marketing, vol. 23, $\mathrm{n}^{\circ}$ 4, pp. 249-264.

PRIETO, M. (2011). «Los estudios sobre turismo en Ecuador». En Espacios en disputa: el turismo en Ecuador (FLACSO, Se). Quito: Mercedes Prieto (coord.).

RAZETO, A. (2013): «Potenciando el desarrollo local de comunidades afectadas por desastres», Revista INVI, vol. XXVII, n 77, pp. 111-136.

RED CIMAS. (2015). Metodologías participativas. Sociopraxis para la creatividad social. (Dextra Ed.). Madrid.

RODRÍGUEZ, M. (2007): «La problemática del riesgo en los proyectos de infraestructura y los contratos internacionales de construcción», Revista e-Mercadotecnia, vol. VI, $\mathrm{n}^{\circ}$ 1, pp. 1-29.

RODRÍGUEZ-TOUBES MUÑIZ, D. y FRAIZ BREA, J.A. (2011). «Gestión proactiva de crisis en el turismo: una experiencia de estudio en Galicia». Pasos. Revista de Turismo y Patrimonio Cultural, vol. 9, no 2, pp. 279-289.

ROMERO, B. (2016): «Sector turístico, buscando una luz al final del túnel», Revista Gestión, n 265, pp. 20-27.

SALVADOR, P. y RAMOS, S. (2010): «100 casos de derecho de daños», InDret, $\mathrm{n}^{\circ} 41$, pp. 38-41.

SERRANO, A. L., FREIRE-CHAGLLA, S., ESPINOZA-FIGUEROA, F., SANMARTÍN, I., FARFÁN, K y VILLAFUERTE, E. (2018). Boletín de Indicadores Turísticos del Cantón Cuenca (Edifepp). Quito.

SOSA, A. y CAZAL, A. (2014): «El espacio público en la ciudad de Cancún frente al proyecto turístico», Revista de Estudios Urbanos y Ciencias Sociales, vol. 5, n² 2, pp. 65-80.

UNWTO. (2008). International recommendations for tourism statistics 2008 (UN). Madrid/New York.

VALENZUELA, E. y COLL, A. (2010): «La construcción y evolución del espacio turístico en Acapulco», Anales de Geografía, vol. XXX, n 1, pp. 163-190. 
VELASCO GONZÁLEZ, M. (2016). «Entre el poder y la racionalidad: gobierno del turismo, política turística, planificación turística y gestión pública del turismo». Pasos. Revista de Turismo y Patrimonio Cultural, vol. 14, nº 3, pp. 577-594.

VILORIA, N. (2012): «Desarrollo turístico y su relación con el transporte», Gestión Turística, $\mathrm{n}^{\circ} 17$, pp. 23-36.

VIVES, X. (2007): «El aeropuerto en la globalización», La Vanguardia, $\mathrm{n}^{\circ} 12$.

WALLINGRE, N. (2005): «La necesidad de implementar una cultura organizacional innovadora en las Pymes hoteleras de Argentina», Tiempo de Gestión, pp. 84-93. 
